\title{
The attitude of Polish rheumatology patients towards telemedicine in the age of the COVID-19 pandemic
}

\author{
Aleksandra Opinc, Zuzanna Łukasik, Joanna Makowska \\ Department of Rheumatology, Medical University of Łódź, Poland
}

\begin{abstract}
Objectives: In response to the outbreak of the SARS-CoV-2 coronavirus pandemic, the Polish government has enabled specialist teleconsultations. Due to this, Polish patients have gained access to continuation of outpatient specialist healthcare using information and communication technologies. The goal of the study was to recognize the needs and expectations as well as the main concerns of Polish rheumatology patients in regard to teleconsultations.

Material and methods: An online-based questionnaire comprising 17 single choice and multiple choice, open-end questions was collected among Polish rheumatology patients directly after the introduction of specialist teleconsultations.

Results: 244 respondents completed the survey. Mean age of the respondents was $40.6 \pm 10.5$ and $92.6 \%$ of them were female. $48 \%$ of the respondents lived not further than 20 kilometres from their outpatient rheumatology clinic. The mean severity of current symptoms, assessed by patients on a visual-analogue scale, was $4.9 \pm 2.4$. 82\% of the respondents selected telephone consultations as the most convenient form of receiving rheumatology advice. The patients highlighted the lack of physical examination (43\%) and additional tests (43.9\%) as the factors discouraging them from teleconsultations; $8.2 \%$ of respondents were against maintaining rheumatology teleconsultations after the SARS-CoV-2 pandemic; 3.7\% of the respondents received information on teleconsultations from a medical professional.

Conclusions: The community of Polish rheumatology patients is eager to benefit from specialist teleconsultations. Telephone consultations are the most eagerly chosen form of remote consultations. Medical professionals should actively promote this form of patient in order to reach the patients who do not use the computer readily.
\end{abstract}

Key words: telerheumatology, telemedicine, rheumatology, teleconsultation, telehealth.

\section{Introduction}

The outbreak of the severe acute respiratory syndrome coronavirus 2 (SARS-CoV-2) pandemic has imposed new challenges on healthcare systems globally. In order to limit infection transmissions measures such as suspension of schooling activities and introduction of e-learning, home offices and temporary closure of sport facilities and shopping centres were introduced in Poland by governmental ordinance. Many healthcare units have restrained their services by suspending planned hospitalizations and limiting the work of outpatient clinics.
In this particular time, it is expected for patients with rheumatic diseases to seek medical advice. The subject of immunosuppressive treatment continuation and the risk of SARS-CoV-2 infection is only one of the commonly raised issues. International societies such as the European League Against Rheumatism (EULAR) as well as local authorities including Polskie Towarzystwo Reumatologiczne (PTR) reacted early by publishing general recommendations for rheumatic patients [1].

As the situation develops dynamically, medical professionals working in the field of rheumatology strive towards identifying the clinical challenges distinct for the

Address for correspondence:

Joanna Makowska, Department of Rheumatology, Medical University of Łódź, 30 Pieniny St., 92-115 Łódź, Poland,

e-mail: joanna.makowska@umed.lodz.pl

Submitted: 30.03.2020; Accepted: 2.06.2020 
speciality, but also share their experiences in providing healthcare services [2, 3].

It is clear that individual patient care cannot be discontinued for the unpredictable period of the pandemic duration, forcing healthcare units to introduce new means of communication and medical counselling.

One such measure is telemedicine, defined broadly by the World Health Organization, which values its role at every stage of providing health services including stating diagnosis, implementing and maintaining treatment, imposing preventive measures, conducting medical research and facilitating education [4].

Until now, telemedicine has not been widely exploited by Polish healthcare providers. On the $11^{\text {th }}$ of March 2020 , in response to the developing epidemiological situation, the Polish National Health Fund (Narodowy Fundusz Zdrowia) enabled specialist teleconsultations to secure the safe continuity of patient care [5]. Previously, telemedical consultations were limited only to some of the medical specialities such as cardiology or geriatrics [6].

The need to implement telemedical consultations to the diagnostic and therapeutic process in rheumatology has been already highlighted by Szpakowski et al. [7] The authors performed a quantitative and qualitative analysis of an internet forum, revealing high interest in rheumatology inquiries yet a low level of discussion. Patients most often seek information on arthralgia and joint swelling, the diagnostic process, interpretation of laboratory tests and managing the symptoms [7].

Considering the effectiveness of specialist teleconsultations in other medical specialties, such as psychiatry [8], telerheumatology emerges as a promising tool to facilitate diagnosis and long-term care.

The aim of the study was to analyse the altitude of Polish patients towards telemedical consultations as well as to evaluate their needs and concerns associated with telerheumatology. As long-term medical care in each specialty requires different measures to assess and control disease activity, we addressed our survey to rheumatic patients specifically.

\section{Material and methods}

A self-designed online survey with convenience (availability) sampling was conducted between $15^{\text {th }}$ of March 2020 and $19^{\text {th }}$ of March 2020, directly after the introduction of specialist teleconsultations in Poland. The total number of questions was 17.

The questionnaire consisted of single and multiple choice with open-end questions. The questionnaire covered respondents' characteristics, patients' attitude to traditional medical care in outpatient healthcare units and to telerheumatology. The questionnaire was created in
Polish and distributed among Polish rheumatic patients, owing to the cooperation with online rheumatic patients' support groups and associations. The full questionnaire is presented as the online supplementary material.

The statistical software used was R (R Core Team, 2017). Descriptive statistics techniques were used for data analysis. An independent sample t-test was performed for comparisons between respondents' subgroups.

\section{Results}

The online survey has been completed by 244 respondents. Most of them were rheumatology patients $(n=234$, $96 \%$ ), 9 of the respondents (3.7\%) were relatives of rheumatology patients, and 1 respondent (0.4\%) reported being both a patient and a relative of a patient.

Mean age of the respondents was $40.6 \pm 10.5$ and the vast majority were females (92.6\%). In $48 \%$ of cases the rheumatology outpatient clinic was located in the same town or not further than 20 kilometres from the place of residence, while in $13.5 \%$ of patients the distance to the rheumatology clinic exceeded 100 kilometres. $60.7 \%$ of patients were attending 2-4, 30.3\% were attending none or a single one, while $9.0 \%$ of them were attending 5 of more different outpatient clinics (Table I).

Various types of arthritis such as rheumatoid arthritis, psoriatic arthritis, ankylosing spondylitis or reactive arthritis were the most common diagnoses and were diagnosed in $65.2 \%$ of respondents. Connective tissue diseases such as systemic lupus erythematous, scleroderma, dermatomyositis, polymyositis or vasculitis were diagnosed in $24.6 \%$ of answerers.

Non-inflammatory diseases (osteoarthritis, gout and osteoporosis) were reported by only one of the respondents (0.4\%). In $5.7 \%$ of respondents no diagnosis was stated, yet they reported the presence of symptoms possibly indicating a rheumatic disease. Mean value of current severity of symptoms was estimated by the patients on the visual analogue scale as $4.9 \pm 2.4$.

In regard to traditional outpatient clinic consultation, the respondents value the possibility of direct conversation with the doctor (70.1\% of respondents), physical examination by a doctor (60.7\%) and the possibility to perform additional tests, e.g. ultrasound examination, blood tests (60.3\%) most. For $41.4 \%$ of the respondents the possibility of consulting the results of additional tests was also important.

Accordingly, the lack of possibility to perform additional tests (such as ultrasound examination, blood tests) as well as to be physically examined by the rheumatologist were the most frequently reported concerns associated with teleconsultations, reported by $43.9 \%$ and $43 \%$ of respon- 
Table I. Demographical data of the study group

\begin{tabular}{|lc|}
\hline Respondents' demographics & $n(\%)$ \\
\hline \begin{tabular}{l} 
Gender \\
\hline Female
\end{tabular} & $226(92.6)$ \\
\hline Male & $18(7.4)$ \\
\hline Age (years) & $40.6 \pm 10.5$ \\
\hline Education & $4(1.6)$ \\
\hline Brimary & $18(7.4)$ \\
\hline Secondary & $86(33.2)$ \\
\hline Higher & $136(55.7)$ \\
\hline Place of residence & $80(32.8)$ \\
\hline City of 200 000 citizens or above & $114(46.7)$ \\
\hline Smaller towns & $50(20.5)$ \\
\hline Rural areas & $116(48)$ \\
\hline \begin{tabular}{l} 
Distance to the outpatient clinic \\
\hline$<20$ km
\end{tabular} & $94(38.5)$ \\
\hline $20-100 \mathrm{~km}$ & $34(13.5)$ \\
\hline$>100 \mathrm{~km}$ & \\
$n-$ number of respondents. &
\end{tabular}

dents respectively. The full list of concerns mentioned by the respondents is presented in Table II.

The most common inquiries to the rheumatologist during teleconsultations would concern advice on how to cope with infections (69.3\% respondents), novel symptoms (61.1\%) and exacerbation of usual ailments (58.6\%). Patients would also seek advice on drug dosage modification (55.3\%) and continuation of chronic therapies $(52.5 \%)$
As for the form of telecommunication, the vast majority of the patients (82.0\%) would prefer telephone consultations, half of the respondents would appreciate the possibility to contact their doctor via e-mail, and 30.3\% of respondents declared interest in video consultations. Only $2.9 \%$ of the respondents would require help from their relatives while participating in telecommunications.

The majority of the patients found out about the possibility of rheumatology teleconsultation via the Internet (71.3\%) or from the other patients (18.0\%), relatives and friends (4.9\%). Only $3.7 \%$ of the respondents were informed about teleconsultations by their rheumatologist or general practitioner.

The vast majority of the responders were of the opinion that teleconsultations should be available also after the end of the SARS-CoV-2 pandemic (88.5\%). In contrast, $8.2 \%$ were against such an idea. The list of reasons to maintain teleconsultations at the rheumatology outpatient clinic, stated by respondents, is presented in Table III.

Statistical analysis showed no differences in patients' approach to telerheumatology when compared between groups defined on the demographic details, distance to outpatient clinic, self-assessed disease activity, or fear of healthcare-acquired infection.

\section{Discussion}

The current epidemiological situation is a challenge for healthcare systems that forces medical professionals to seek and implement novel solutions. Telemedicine is becoming an indispensable and, most of all, a safer tool for maintaining the continuity of patient care. Most of the publications or conference proceedings on telemed-

Table II. Concerns associated with teleconsultations with rheumatologist, stated by the respondents

\begin{tabular}{|c|c|c|}
\hline Concerns associated with rheumatology teleconsultation & $n$ & $\%$ \\
\hline Lack of possibility to perform additional tests such as ultrasound examination, blood tests & 107 & 43.9 \\
\hline Lack of physical examination, performed by the doctor & 105 & 43 \\
\hline Concern whether the doctor would understand my complaints & 51 & 20.9 \\
\hline Concern that the patient would not accurately present his/her symptoms & 74 & 30.3 \\
\hline Concern that the doctor would not have enough time for each patient & 57 & 23.4 \\
\hline Weak computer skills & 1 & 0.4 \\
\hline Lack of access to equipment (computer, telephone) enabling teleconsultations & 2 & 0.8 \\
\hline Concern whether the patients would get a prescription & 32 & 13.1 \\
\hline Concern whether the patient would hear the doctor, hearing impairment & 2 & 0.8 \\
\hline No concerns associated with teleconsultations & 57 & 23.4 \\
\hline No intentions to participate in teleconsultations & 4 & 1.6 \\
\hline Other concerns & 8 & 3.3 \\
\hline
\end{tabular}

$n$ - number of respondents. 
Table III. List of reasons to maintain teleconsultations at the rheumatology outpatient clinic after SARS-CoV-2 pandemic, stated by respondents

\begin{tabular}{|c|c|c|}
\hline Reasons to maintain telecommunication as a form of appointment at the rheumatology outpatient clinic & $n$ & $\%$ \\
\hline Walking difficulties & 25 & 10.3 \\
\hline Telecommunications are a more convenient form than direct appointments & 98 & 40.2 \\
\hline Limited transport options to the rheumatology outpatient clinic & 33 & 13.5 \\
\hline Concerns about getting infected & 85 & 34.8 \\
\hline Too long waiting time for the appointment & 128 & 52.5 \\
\hline Possibility to shorten the queues to the outpatient clinic & 153 & 62.7 \\
\hline Telecommunication at the rheumatology outpatient clinic should not be maintained after the pandemic & 20 & 8.2 \\
\hline Other reasons & 9 & 3.7 \\
\hline
\end{tabular}

$n$-number of respondents.

icine in Poland focused on selected disciplines such as family medicine, cardiology, orthopaedics, radiology and pathology [9].

Information and communication technologies were mostly used for disease activity monitoring, as a means of physical activity encouragement or as user-centred applications for disease management. Our study demonstrated a positive attitude to telerheumatology among Polish rheumatology patients, a group confronted with the possibility of specialist teleconsultations for the first time. The vast majority of the respondents declared the wish to maintain teleconsultations even after the pandemic.

Rheumatic diseases are usually chronic and progressive, requiring a long-term partnership between the patient and the doctor [10]. Most of the rheumatology outpatient clinics are located in urban areas, which limits the access to specialist care to the patients from suburban or rural areas. Telemedicine could then serve an auxiliary role in the management of chronic diseases, in particular in the follow-up of the patients with lower access to outpatient clinics.

In a questionnaire-based survey addressed to rheumatology patients living in Australian areas remote from rheumatology clinics, the majority of the patients appreciated it as a money- and timesaving form of medical advice (respectively $85.7 \%$ and $89.3 \%$ of the respondents). Moreover, $63 \%$ of patients stated that they would prefer to receive specialist advice via teleconsultations over travelling a long distance to traditional appointments [11].

However, in our study we did not observe any differences in patients' attitude towards teleconsultations between the groups of patients living in the biggest cities vs rural areas, nor patients living close to and far from their rheumatology outpatient clinic. According to the literature, as many as $90 \%$ of respondents considered their teleconsultation as satisfactory [11].
Similarly, in a study by Kulcsar et al. [12], 2 out of 3 patients stated that the care obtained during the telemedical consultation was comparable to direct appointment, with more than half of the respondents willing to use telerheumatology again. However, only $25 \%$ of respondents were of the opinion that telerheumatology is more convenient than a face-to face visit [12].

By contrast, in our study $40.16 \%$ of respondents considered teleconsultation as a more convenient form. Since so far telerheumatology has not been commonly used in Poland, we focused on the attitude of the patients towards telemedical counselling and no inquiries over the satisfaction from previous teleconsultations were made.

Physical examination with the assessment of joint inflammation, mobility and cutaneous lesions remains a necessary step in the diagnostic process in rheumatology [13]. Establishing a diagnosis and implementing initial therapy require physical examination and, in many cases, also additional tests. These remain impossible to perform during a telemedical appointment.

In the study by Graham et al. [14], the majority of patients were improperly diagnosed by means of telerheumatology, as only $35 \%$ of diagnoses were stated correctly over the telephone and $40 \%$ by video-phone consultation, with no significant difference in the accuracy between telephone and video-phone consultation. Moreover, most cases of joint swelling or deformation as well as cutaneous or nail lesions remained undetected if assessed with telerheumatology.

According to Leggett et al. [15], the diagnostic accuracy in rheumatology patients was poor as compared to direct examination if the consultation was performed only by means of telephone, yet improved significantly if two-way video telecommunications were used.

In our study, the majority of respondents selected telephone consultations as the most convenient means 
of contact, while only one in three respondents opted for video consultations.

On the other hand, Nguyen-Oghalai et al. [16], achieved $79 \%$ accuracy in the diagnoses made during telemedical consultations as compared to direct appointments. Moreover, all of the patients with inflammatory rheumatic diseases were identified by means of telerheumatology.

Our department provides rheumatology teleconsultations only as a continuation of patient care. Our experience gained so far suggests that consultations via telephone correspond to medical interview, excluding the crucial diagnostic element of physical examination. The accuracy of that anamnesis increases with the duration of the disease. Accordingly, a study from Denmark proved tele-health follow-up of rheumatoid arthritis patients as similarly effective to conventional outpatient follow-up for disease activity control [17].

Our study was based on an online survey with convenience sampling, which is associated with a significant risk of selection bias and limits the credibility of our study. We can conclude that it was completed by patients with at least basic computer skills. Patients with the access and ability to use computers could constitute a preselected group willing to benefit from novel technologies including telemedical consultations. Furthermore, the mean age of our respondents was relatively low, which could impact the results.

Younger generations are more familiar with novel technologies, and therefore could be more prone to seek telemedical advice. Performing such a questionnaire with the patients registered in an exemplary rheumatology outpatient clinic via the telephone could bring more representative answers.

The study by Ferucci et al. [18], however, excluded demographic factors as associated with telemedicine use among rheumatoid arthritis patients. Higher disease activity and higher number of rheumatologist visits in the preceding year were found to distinguish the patients with a positive attitude to telerheumatology [18].

The majority of the patients stated that they would seek medical advice on how to cope with infections via telecommunication. However, we cannot exclude the possibility that this trend was influenced by the current epidemiological situation.

Notably, the majority of the patients learnt about the possibility of teleconsultations via the Internet or from the other patients, while only a few of them were informed by their rheumatologist or general practitioner. This points towards the need for medical professionals to actively inform their patients about novel possibilities and convince them to try telemedical consultations. The newly implemented healthcare strategies and communication platforms created in the time of the COVID-19 pandemic are likely to remain when the pandemic has passed [19].

Medical professionals working in the field of rheumatology across the world are making utmost efforts to maintain high quality, specialist patient care in the time of the COVID-19 pandemic. Regardless of the geographical area, elective hospitalizations and non-urgent diagnostics have been postponed and healthcare systems globally are facing the same challenges. Continuation of therapy with biologicals is one of them [20].

Despite early recommendations from local and international rheumatology consortiums advising not to discontinue therapy with biological disease modifying antirheumatic drugs, many patients express concern regarding their immunosuppressive therapy [21]. This issue can easily be clarified by means of telemedicine. However, these drugs are often administered at specialized clinics and the safety and efficacy of treatment are strictly controlled.

In Australia, a temporary change in law has allowed for streamlined approval of biologics by telephone [22]. The rheumatological community is eagerly sharing experiences and successfully implemented novel solutions.

Table IV. Summarized key points of this study

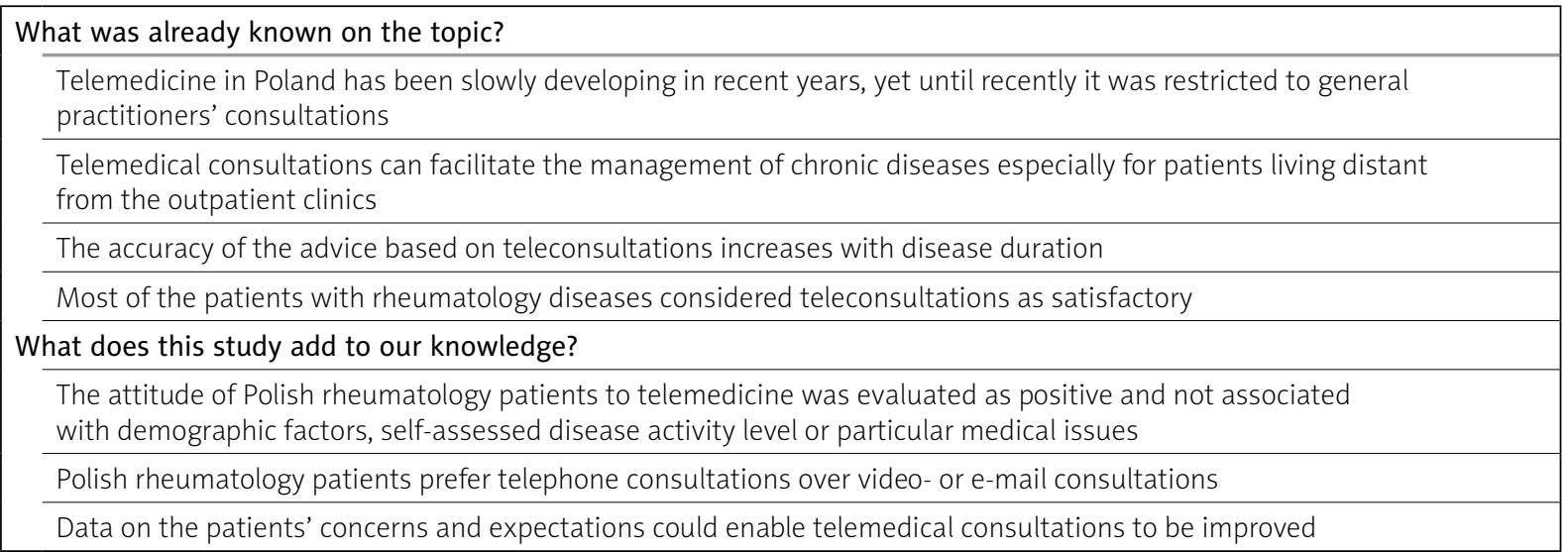




\section{Conclusions}

The rapidly developing epidemiological situation has forced the implementation of specialist teleconsultations in Poland.

Our online-based questionnaire (Appendix 1) shows that the community of Polish patients with rheumatic diseases is in favour of receiving rheumatology advice through the use of information and communication technologies, in particular via telephone and e-mail.

The respondents' attitude towards telerheumatology was not associated with any demographic factors, self-assessed disease activity level or particular medical issues. The collected conclusions from our study are presented in Table IV.

The Internet was the main source of information on teleconsultations, suggesting that medical professionals should actively promote this form of patient care in order to reach those patients who do not use the computer readily.

The authors declare no conflict of interest.

\section{References}

1. Stajszczyk M, Brzosko M. Statement of the Polish Society of Rheumatology and the national consultant in the field of rheumatology to patients and physicians in connection with the infection COVID-19. Available at: http://www.reumatologia.ptr. net.pl/?update-aktualizacja-komunikat-polskiego-towarzystwa-reumatologicznego-i-konsultanta-krajowego-w-dziedzinie-reumatologii-dla-pacjentow-i-lekarzy-w-zwiazku-z-szerzaca-sie-infekcja-covid-19,291 (access: 21.04.2020).

2. Perricone C, Gerli R. Being a rheumatologist and a patient with a rheumatic disease today: a perspective at the time of COVID-19. Eur J Rheumatol 2020, DOI: 10.5152/eurjrheum.2020.2057 [Epub ahead of print].

3. Misra DP, Agarwal V, Gasparyan AY, Zimba O. Rheumatologists' perspective on coronavirus disease 19 (COVID-19) and potential therapeutic targets. Clin Rheumatol 2020, DOI: 10.1007/ s10067-020-05073-9 [Epub ahead of print].

4. Ryu S. Telemedicine: opportunities and developments in member states: report on the second global survey on eHealth 2009 (Global Observatory for eHealth Series, Volume 2) Healthc Inform Res 2012; 18: 153-155, DOI: 10.4258/hir.2012.18.2.153

5. Attachment $1 \mathrm{a}$ and $1 \mathrm{c}$ to the Minister of the National Health Fund Ordinance on the implementation of contracts for the provision of healthcare services such as outpatient specialist care number 182/2019/DSOZ. Available at: https://www.nfz. gov.pl/zarzadzenie (access: 26.03.2020).

6. Amendment to the Act on the information system in health care number 63/2015/DSOZ. Available at: https://www.nfz. gov.pl/zarzadzenia-prezesa/zarzadzenia-prezesa-nfz/zarzadzenie-nr-632015dsoz,6410.html (access: 21.04.2020).

7. Szpakowski R, Maślińska M, Dykowska G, Zając P. Analysis of information on rheumatology from a selected Internet forum in the context of the need for telemedicine solutions. Reumatologia 2015; 53: 260-267, DOI: 10.5114/reum.2015.55829.

8. Krzystanek M, Borkowski M, Krysta K. Psychiatry as a leader of contemporary telemedicine in Poland. Reumatologia 2018; 56: 65-66, DOI: 10.5114/reum.2018.75515.

9. Glinkowski WM, Karlińska M, Karliński M, Krupiński EA. Telemedicine and eHealth in Poland from 1995 to 2015. Adv Clin Exp Med 2018; 27: 277-282, DOI: 10.17219/acem/74124.

10. Nikiphorou E, Alunno A, Carmona L, et al. Patient-physician collaboration in rheumatology: a necessity, RMD Open 2017; 3: e000499, DOI: 10.1136/rmdopen-2017-000499.

11.Poulsen KA, Millen CM, Lakshman UI, et al. Satisfaction with rural rheumatology telemedicine service. Int J Rheum Dis 2015; 18: 304-314, DOI: 10.1111/1756-185X.12491.

12. Kulcsar Z, Albert D, Ercolano E, Mecchella JN. Telerheumatology: a technology appropriate for virtually all. Semin Arthritis Rheum 2016; 46: 380-385, DOI: 10.1016/j.semarthrit.2016.05.013.

13. Castrejõn I, McCollum L, Tanriover MD, Pincus T. Importance of patient history and physical examination in rheumatoid arthritis compared to other chronic diseases: results of a physician survey. Arthritis Care Res (Hoboken) 2012; 64: 1250-1255, DOI: 10.1002/acr.21650.

14. Graham LE, McGimpsey S, Wright S, et al. Could a low-cost audio-visual link be useful in rheumatology? J Telemed Telecare 2000; 6 (Suppl 1): 35-37, DOI: 10.1258/1357633001934078.

15. Leggett PF, Graham L, Steele K, et al. Telerheumatology diagnostic accuracy and acceptability to patient, specialist, and general practitioner. Br J Gen Pract 2001; 51: 746-748.

16. Nguyen-Oghalai TU, Hunter K, Lyon M. Telerheumatology: the VA experience. South Med J 2018; 111: 359-362, DOI: 10.14423/SMJ.0000000000000811.

17. de Thurah A, Stengaard-Pedersen K, Axelsen M, et al. Telehealth follow-up strategy for tight control of disease activity in rheumatoid arthritis: results of a randomized controlled trial. Arthritis Care Res (Hoboken) 2018; 70: 353-360, DOI: 10.1002/ acr.23280

18. Ferucci ED, Holck P, Day GM, et al. Factors associated with use of telemedicine for follow-up of rheumatoid arthritis. Arthritis Care Res (Hoboken) 2019, DOI: 10.1002/acr.24049 [Epub ahead of print].

19. Lewandowski LB, Hsieh E. Global rheumatology in the time of COVID-19. Lancet Rheumatol 2020; 2: e254-e255, DOI: 10.1016/S2665-9913(20)30091-6.

20. Rheumatology patients treatment jeopardized by coronavirus pandemic. https://www.globaldata.com/rheumatologypatients-treatment-jeopardized-by-coronavirus-pandemic/.

21. Kirby T. Rheumatologists rapidly adjust patient care during COVID-19 pandemic. Lancet Rheumatol 2020; 2: e258, DOI: 10.1016/s2665-9913(20)30094-1.

22. Cai K, He J, Wong PK, Manolios N. The impact of COVID-19 on rheumatology clinical practice and university teaching in Sydney, Australia. Eur J Rheumatol 2020, DOI: 10.5152/eurjrheum.2020.20060 [Epub ahead of print]. 


\section{Appendix 1}

\section{The questionnaire sent to rheumatology patients}

1. Telerheumatology is a subject of importance to me because:

- I am a rheumatology patient

- I am a relative of a rheumatology patient

- Other

2. Gender:

- Male

- Female

3. Age:

4. Education:

- Primary

- Basic vocational

- Secondary

- Higher

5. Place of residence:

- city $>200000$ inhabitants

- city 100-200 000 inhabitants

- city 20-100 000 inhabitants

- town 5-20 000 inhabitants

- village or town $<5000$ inhabitants

6. How far from your rheumatology outpatient clinic do you live?

- My rheumatology outpatient clinic is located in the same town where I live

- Less than $20 \mathrm{~km}$

- Between 20 and $50 \mathrm{~km}$

- Between 50 and $100 \mathrm{~km}$

- More than $100 \mathrm{~km}$ away

7. Have you been diagnosed with a rheumatic disease?

- Yes, with an inflammatory rheumatic disease (for example, rheumatoid arthritis, psoriatic arthritis, axial spondyloarthropathy)

- Yes, with a non-inflammatory rheumatic disease (for example, osteoarthritis, gout, osteoporosis)

- Yes, with a connective tissue disease (systemic lupus erythematosus, systemic sclerosis, dermatomyositis, polymyositis)

- Yes, a different rheumatic disease

- No, but I suffer from symptoms suggesting a rheumatic disease

- No, but I need rheumatology advice for another reason

- Other

8. Please, assess your current complaints associated with the rheumatic disease on a scale from 1 to 10 :

No ailment Symptoms disabling everyday functioning

$\begin{array}{llllllllllll}0 & 1 & 2 & 3 & 4 & 5 & 6 & 7 & 8 & 9 & 10\end{array}$

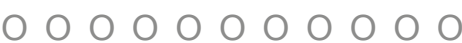

9. What do you value most in the direct contact with your rheumatologist during traditional outpatient clinic consultations? Multiple choice question.

- The direct talk with my rheumatologist

- The possibility of physical examination (palpation, auscultation) performed by the doctor

- The possibility of undergoing additional tests (such as ultrasound examination, tests from blood)

- The possibility to show your test results to the doctor

- Other

10. Which information and communication technologies do you find best to contact with your rheumatologist? Multiple choice question.

- Phone

- Online communicators such as Skype

- E-mail

- I am not interested in receiving medical advice using information and communication technologies

- Other 
11. Which of the possible issues could affect your rheumatology teleconsultation? Multiple choice question.

- Lack of the possibility to perform additional tests such as ultrasound examination, or tests from blood

- Lack of physical examination performed (palpation, auscultation) by the doctor

- Concern about the doctor's understanding of my complaints

- Concern about not being able to accurately present my symptoms during a teleconsultation

- Concern about the doctor not having enough time for me during a teleconsultation

- Problems with using the computer

- Lack of access to the equipment enabling teleconsultations (computer, telephone)

- Concern about receiving drug prescriptions

- Concern about following the teleconsultation due to hearing impairment

- I have no concerns associated with teleconsultations

- I have no intention to participate in teleconsultations

- Other

12. On which medical issues associated with your rheumatic disease would you like to receive advice during a teleconsultation? Multiple choice question.

- Advice on medication dosage modification

- Receiving prescription on my current medication

- Advice in case of an infection

- Advice in case of aggravation of my usual symptoms

- Advice in case of new complaints or symptoms

- Advice in case of side effects of my medication

- I am not interested in receiving medical advice using information and communication technologies

- Other

13. Will you require any help in order to participate in a teleconsultation?

- Yes, I will need help with using the equipment enabling teleconsultations (computer, telephone)

- Yes, I will need help other than with using the computer

- No, I will not need any help

14. How did you learn about the possibility of teleconsultations in a rheumatology outpatient clinic?

- From my rheumatologist

- From my family doctor

- From another healthcare professional

- From the website of my hospital / outpatient clinic

- From other patients

- From my family and friends

- Other

15. Do you think that after the SARS-CoV-2 pandemic rheumatology teleconsultations should be continued?

- Definitely yes

- Yes

- Neither agree nor disagree

- No

- Definitely not

16. Please select all the reasons for which you would want rheumatology teleconsultations to be continued after the SARS-CoV-2 pandemic:

- I have difficulties walking

- Teleconsultations are more convenient to me than a traditional outpatient clinic consultation

- I have limited possibilities of travelling to my outpatient clinic

- I am afraid of infections

- The waiting time for a traditional consultation is too long

- Teleconsultations could shorten the queues to healthcare professionals

- I think that teleconsultations should not be continued after the SARS-CoV-2 pandemic

- Other

17. Please select the number of the specialist outpatient clinics you attend:

- 1

- Between 2 and 4

- More than 5 